\title{
Conway's Games and Some of their Basic Properties
}

\author{
Robin Nittka \\ Institute of Applied Analysis \\ University of Ulm \\ 89069 Ulm, Germany
}

\begin{abstract}
Summary. We formulate a few basic concepts of J. H. Conway's theory of games based on his book [6]. This is a first step towards formalizing Conway's theory of numbers into Mizar, which is an approach to proving the existence of a FIELD (i.e., a proper class that satisfies the axioms of a real-closed field) that includes the reals and ordinals, thus providing a uniform, independent and simple approach to these two constructions that does not go via the rational numbers and hence does for example not need the notion of a quotient field.

In this first article on Conway's games, we provide a definition of games, their birthdays (or ranks), their trees (a notion which is not in Conway's book, but is useful as a tool), their negates and their signs, together with some elementary properties of these notions. If one is interested only in Conway's numbers, it would have been easier to define them directly, but going via the notion of a game is a more general approach in the sense that a number is a special instance of a game and that there is a rich theory of games that are not numbers.

The main obstacle in formulating these topics in Mizar is that all definitions are highly recursive, which is not entirely simple to translate into the Mizar language. For example, according to Conway's definition, a game is an object consisting of left and right options which are themselves games, and this is by definition the only way to construct a game. This cannot directly be translated into Mizar, but a theorem is included in the article which proves that our definition is equivalent to Conway's.
\end{abstract}

MML identifier: $\underline{\text { CGAMES_1 }}$, version: $\underline{7.11 .07 \quad 4.156 .1112}$

The terminology and notation used here have been introduced in the following articles: [1], [4], [7], [5], [2], [3], [9], and [8]. 


\section{Construction of Days}

We follow the rules: $x, z, s$ are sets, $\alpha, \beta$ are ordinal numbers, and $n$ is a natural number.

We introduce lefts-rights which are systems

$\langle$ left options, right options >,

where the left options and the right options constitute sets.

The functor $\mathbf{0}$ is defined by:

(Def. 1) $\mathbf{0}=\langle\emptyset, \emptyset\rangle$.

One can verify that there exists a left-right which is strict.

Let us consider $\alpha$. The functor ConwayDay $\alpha$ yields a set and is defined by the condition (Def. 2).

(Def. 2) There exists a transfinite sequence $f$ such that $\alpha \in \operatorname{dom} f$ and $f(\alpha)=$ ConwayDay $\alpha$ and for every $\beta$ such that $\beta \in \operatorname{dom} f$ holds $f(\beta)=\{\langle x, y\rangle$ : $x$ ranges over subsets of $\bigcup \operatorname{rng}(f\lceil\beta), y$ ranges over subsets of $\bigcup \operatorname{rng}(f\lceil\beta)\}$.

We now state three propositions:

(1) $z \in$ ConwayDay $\alpha$ if and only if there exists a strict left-right $w$ such that $z=w$ and for every $x$ such that $x \in$ (the left options of $w) \cup$ (the right options of $w$ ) there exists $\beta$ such that $\beta \in \alpha$ and $x \in$ ConwayDay $\beta$.

(2) ConwayDay $0=\{\boldsymbol{0}\}$.

(3) If $\alpha \subseteq \beta$, then ConwayDay $\alpha \subseteq$ ConwayDay $\beta$.

Let us consider $\alpha$. Note that ConwayDay $\alpha$ is non empty.

\section{GAMES}

Let us consider $x$. We say that $x$ is Conway game-like if and only if:

(Def. 3) There exists $\alpha$ such that $x \in$ ConwayDay $\alpha$.

Let us consider $\alpha$. Note that every element of ConwayDay $\alpha$ is Conway gamelike.

Let us observe that $\mathbf{0}$ is Conway game-like.

One can check that there exists a left-right which is Conway game-like and strict and there exists a set which is Conway game-like.

A Conway game is a Conway game-like set.

$\mathbf{0}$ is an element of ConwayDay 0 .

The element 1 of ConwayDay 1 is defined by:

(Def. 4) $\mathbf{1}=\langle\{\mathbf{0}\}, \emptyset\rangle$.

The element $*$ of ConwayDay 1 is defined as follows:

(Def. 5) $*=\langle\{\mathbf{0}\},\{\mathbf{0}\}\rangle$.

In the sequel $g, g_{0}, g_{1}, g_{2}, g_{3}, g_{4}, g_{5}, g_{6}$ are Conway games.

We now state the proposition 
(4) $g$ is a strict left-right.

One can verify that every left-right which is Conway game-like is also strict.

Let us consider $g$. The left options of $g$ is defined as follows:

(Def. 6) There exists a left-right $w$ such that $g=w$ and the left options of $g=$ the left options of $w$.

The right options of $g$ is defined by:

(Def. 7) There exists a left-right $w$ such that $g=w$ and the right options of $g=$ the right options of $w$.

Let us consider $g$. The options of $g$ is defined by:

(Def. 8) The options of $g=($ the left options of $g) \cup$ (the right options of $g$ ).

Next we state the proposition

(5) $g_{1}=g_{2}$ if and only if the following conditions are satisfied:

(i) the left options of $g_{1}=$ the left options of $g_{2}$, and

(ii) the right options of $g_{1}=$ the right options of $g_{2}$.

One can verify the following observations:

* the left options of $\mathbf{0}$ is empty,

* the right options of $\mathbf{0}$ is empty, and

* the right options of $\mathbf{1}$ is empty.

Next we state four propositions:

(6) $g=\mathbf{0}$ iff the options of $g=\emptyset$.

(7) $x \in$ the left options of $\mathbf{1}$ iff $x=\mathbf{0}$.

(8)(i) $\quad x \in$ the options of $*$ iff $x=\mathbf{0}$,

(ii) $\quad x \in$ the left options of $*$ iff $x=\mathbf{0}$, and

(iii) $\quad x \in$ the right options of $*$ iff $x=\mathbf{0}$.

(9) $g \in$ ConwayDay $\alpha$ iff for every $x$ such that $x \in$ the options of $g$ there exists $\beta$ such that $\beta \in \alpha$ and $x \in$ ConwayDay $\beta$.

Let $g$ be a set. Let us assume that $g$ is a Conway game. The functor ConwayRank $g$ yields an ordinal number and is defined as follows:

(Def. 9) $g \in$ ConwayDay ConwayRank $g$ and for every $\beta$ such that $\beta \in$ ConwayRank $g$ holds $g \notin$ ConwayDay $\beta$.

One can prove the following propositions:

(10) If $g \in$ ConwayDay $\alpha$ and $x \in$ the options of $g$, then $x \in$ ConwayDay $\alpha$.

(11) If $g \in$ ConwayDay $\alpha$ and if $x \in$ the left options of $g$ or $x \in$ the right options of $g$, then $x \in$ ConwayDay $\alpha$.

(12) $g \in$ ConwayDay $\alpha$ iff ConwayRank $g \subseteq \alpha$.

(13) ConwayRank $g \in \alpha$ iff there exists $\beta$ such that $\beta \in \alpha$ and $g \in$ ConwayDay $\beta$.

(14) If $g_{3} \in$ the options of $g$, then ConwayRank $g_{3} \in$ ConwayRank $g$. 
(15) If $g_{3} \in$ the left options of $g$ or $g_{3} \in$ the right options of $g$, then ConwayRank $g_{3} \in$ ConwayRank $g$.

(16) $g \notin$ the options of $g$.

(17) If $x \in$ the options of $g$, then $x$ is a Conway game-like left-right.

(18) If $x \in$ the left options of $g$ or $x \in$ the right options of $g$, then $x$ is a Conway game-like left-right.

(19) Let $w$ be a strict left-right. Then $w$ is a Conway game if and only if for every $z$ such that $z \in$ (the left options of $w) \cup$ (the right options of $w$ ) holds $z$ is a Conway game.

\section{Schemes of Induction}

In this article we present several logical schemes. The scheme ConwayGameMinTot concerns a unary predicate $\mathcal{P}$, and states that:

There exists $g$ such that $\mathcal{P}[g]$ and for every $g_{1}$ such that ConwayRank $g_{1} \in$ ConwayRank $g$ holds not $\mathcal{P}\left[g_{1}\right]$

provided the following condition is met:

- There exists $g$ such that $\mathcal{P}[g]$.

The scheme ConwayGameMin concerns a unary predicate $\mathcal{P}$, and states that:

There exists $g$ such that $\mathcal{P}[g]$ and for every $g_{3}$ such that $g_{3} \in$ the options of $g$ holds not $\mathcal{P}\left[g_{3}\right]$

provided the parameters satisfy the following condition:

- There exists $g$ such that $\mathcal{P}[g]$.

The scheme ConwayGameInd concerns a unary predicate $\mathcal{P}$, and states that: For every $g$ holds $\mathcal{P}[g]$ provided the following condition is met:

- For every $g$ such that for every $g_{3}$ such that $g_{3} \in$ the options of $g$ holds $\mathcal{P}\left[g_{3}\right]$ holds $\mathcal{P}[g]$.

\section{Tree of a Game}

Let $f$ be a function. We say that $f$ is Conway game-valued if and only if:

(Def. 10) For every $x$ such that $x \in \operatorname{dom} f$ holds $f(x)$ is a Conway game.

Let us consider $g$. One can verify that $\langle g\rangle$ is Conway game-valued.

Let us mention that there exists a finite sequence which is Conway gamevalued and non empty.

Let $f$ be a non empty finite sequence. Observe that every element of $\operatorname{dom} f$ is natural and non empty.

Let $f$ be a Conway game-valued non empty function and let $x$ be an element of $\operatorname{dom} f$. Note that $f(x)$ is Conway game-like. 
Let $f$ be a Conway game-valued non empty finite sequence. We say that $f$ is Conway game chain-like if and only if:

(Def. 11) For every element $n$ of $\operatorname{dom} f$ such that $n>1$ holds $f(n-1) \in$ the options of $f(n)$.

One can prove the following proposition

(20) For every finite sequence $f$ and for every $n$ such that $n \in \operatorname{dom} f$ and $n>1$ holds $n-1 \in \operatorname{dom} f$.

Let us consider $g$. Observe that $\langle g\rangle$ is Conway game chain-like.

Let us observe that there exists a Conway game-valued non empty finite sequence which is Conway game chain-like.

A Conway game chain is a Conway game chain-like Conway game-valued non empty finite sequence.

Next we state three propositions:

(21) For every Conway game chain $f$ and for all elements $n, m$ of $\operatorname{dom} f$ such that $n<m$ holds ConwayRank $f(n) \in$ ConwayRank $f(m)$.

(22) For every Conway game chain $f$ and for all elements $n, m$ of $\operatorname{dom} f$ such that $n \leq m$ holds ConwayRank $f(n) \subseteq$ ConwayRank $f(m)$.

(23) For every Conway game chain $f$ such that $f(\operatorname{len} f) \in$ ConwayDay $\alpha$ holds $f(1) \in$ ConwayDay $\alpha$.

Let us consider $g$. The tree of $g$ yields a set and is defined as follows:

(Def. 12) $z \in$ the tree of $g$ iff there exists a Conway game chain $f$ such that $f(1)=z$ and $f(\operatorname{len} f)=g$.

Let us consider $g$. Observe that the tree of $g$ is non empty.

Let us consider $g$. The proper tree of $g$ yielding a subset of the tree of $g$ is defined by:

(Def. 13) The proper tree of $g=($ the tree of $g) \backslash\{g\}$.

We now state the proposition

(24) $g \in$ the tree of $g$.

Let us consider $\alpha$ and let $g$ be an element of ConwayDay $\alpha$. Then the tree of $g$ is a subset of ConwayDay $\alpha$.

Let us consider $g$. One can verify that every element of the tree of $g$ is Conway game-like.

The following propositions are true:

(25) For every Conway game chain $f$ and for every non empty natural number $n$ holds $f\lceil n$ is a Conway game chain.

(26) Let $f_{1}, f_{2}$ be Conway game chains. Given $g$ such that $g=f_{2}(1)$ and $f_{1}\left(\right.$ len $\left.f_{1}\right) \in$ the options of $g$. Then $f_{1} \frown f_{2}$ is a Conway game chain.

(27) $x \in$ the tree of $g$ iff $x=g$ or there exists $g_{3}$ such that $g_{3} \in$ the options of $g$ and $x \in$ the tree of $g_{3}$. 
(28) If $g_{3} \in$ the tree of $g$, then $g_{3}=g$ or ConwayRank $g_{3} \in$ ConwayRank $g$.

(29) If $g_{3} \in$ the tree of $g$, then ConwayRank $g_{3} \subseteq$ ConwayRank $g$.

(30) For every set $s$ such that $g \in s$ and for every $g_{1}$ such that $g_{1} \in s$ holds the options of $g_{1} \subseteq s$ holds the tree of $g \subseteq s$.

(31) If $g_{1} \in$ the tree of $g_{2}$, then the tree of $g_{1} \subseteq$ the tree of $g_{2}$.

(32) If $g_{1} \in$ the tree of $g_{2}$, then the proper tree of $g_{1} \subseteq$ the proper tree of $g_{2}$.

(33) The options of $g \subseteq$ the proper tree of $g$.

(34) The options of $g \subseteq$ the tree of $g$.

(35) If $g_{1} \in$ the proper tree of $g_{2}$, then the tree of $g_{1} \subseteq$ the proper tree of $g_{2}$.

(36) If $g_{3} \in$ the options of $g$, then the tree of $g_{3} \subseteq$ the proper tree of $g$.

(37) The tree of $\mathbf{0}=\{\mathbf{0}\}$.

(38) $\mathbf{0} \in$ the tree of $g$.

The scheme ConwayGameMin2 concerns a unary predicate $\mathcal{P}$, and states that:

There exists $g$ such that $\mathcal{P}[g]$ and for every $g_{3}$ such that $g_{3} \in$ the proper tree of $g$ holds not $\mathcal{P}\left[g_{3}\right]$

provided the following condition is met:

- There exists $g$ such that $\mathcal{P}[g]$.

\section{Scheme about Definability of Functions by Recursion}

Now we present two schemes. The scheme Func1RecUniq deals with a binary functor $\mathcal{F}$ yielding a set, and states that:

Let given $g$ and $f_{1}, f_{2}$ be functions. Suppose that

(i) $\operatorname{dom} f_{1}=$ the tree of $g$,

(ii) $\operatorname{dom} f_{2}=$ the tree of $g$,

(iii) for every $g_{1}$ such that $g_{1} \in \operatorname{dom} f_{1}$ holds $f_{1}\left(g_{1}\right)=\mathcal{F}\left(g_{1}, f_{1}\right.$ †the proper tree of $g_{1}$ ), and

(iv) for every $g_{1}$ such that $g_{1} \in \operatorname{dom} f_{2}$ holds $f_{2}\left(g_{1}\right)=\mathcal{F}\left(g_{1}, f_{2}\right.$ ¡the proper tree of $g_{1}$ ).

Then $f_{1}=f_{2}$

for all values of the parameter.

The scheme Func1RecEx deals with a binary functor $\mathcal{F}$ yielding a set, and states that:

There exists a function $f$ such that $\operatorname{dom} f=$ the tree of $g$ and for every $g_{1}$ such that $g_{1} \in \operatorname{dom} f$ holds $f\left(g_{1}\right)=\mathcal{F}\left(g_{1}, f\right.$ 个the proper tree of $g_{1}$ )

for all values of the parameter. 


\section{The Negative And Signs}

Let us consider $g$. The functor $-g$ is defined by the condition (Def. 14).

(Def. 14) There exists a function $f$ such that

(i) $\operatorname{dom} f=$ the tree of $g$,

(ii) $-g=f(g)$, and

(iii) for every $g_{1}$ such that $g_{1} \in \operatorname{dom} f$ holds $f\left(g_{1}\right)=\left\langle\left\{f\left(g_{4}\right) ; g_{4}\right.\right.$ ranges over elements of the right options of $g_{1}$ : the right options of $\left.g_{1} \neq \emptyset\right\},\left\{f\left(g_{7}\right) ; g_{7}\right.$ ranges over elements of the left options of $g_{1}$ : the left options of $\left.\left.g_{1} \neq \emptyset\right\}\right\rangle$.

Let us consider $g$. One can check that $-g$ is Conway game-like.

We now state three propositions:

(39)(i) For every $x$ holds $x \in$ the left options of $-g$ iff there exists $g_{4}$ such that $g_{4} \in$ the right options of $g$ and $x=-g_{4}$, and

(ii) for every $x$ holds $x \in$ the right options of $-g$ iff there exists $g_{7}$ such that $g_{7} \in$ the left options of $g$ and $x=-g_{7}$.

(40) $--g=g$.

(41)(i) $\quad g_{3} \in$ the left options of $-g$ iff $-g_{3} \in$ the right options of $g$,

(ii) $g_{3} \in$ the left options of $g$ iff $-g_{3} \in$ the right options of $-g$,

(iii) $\quad g_{3} \in$ the right options of $-g$ iff $-g_{3} \in$ the left options of $g$, and

(iv) $g_{3} \in$ the right options of $g$ iff $-g_{3} \in$ the left options of $-g$.

Let us consider $g$. We say that $g$ is non-negative if and only if the condition (Def. 15) is satisfied.

(Def. 15) There exists $s$ such that

(i) $g \in s$, and

(ii) for every $g_{1}$ such that $g_{1} \in s$ and for every $g_{4}$ such that $g_{4} \in$ the right options of $g_{1}$ there exists $g_{8}$ such that $g_{8} \in$ the left options of $g_{4}$ and $g_{8} \in s$.

Let us consider $g$. We say that $g$ is non-positive if and only if:

(Def. 16) $-g$ is non-negative.

Let us consider $g$. We say that $g$ is zero if and only if:

(Def. 17) $g$ is non-negative and non-positive.

We say that $g$ is fuzzy if and only if:

(Def. 18) $g$ is not non-negative and $g$ is not non-positive.

Let us consider $g$. We say that $g$ is positive if and only if:

(Def. 19) $g$ is non-negative and $g$ is not zero.

We say that $g$ is negative if and only if:

(Def. 20) $g$ is non-positive and $g$ is not zero.

One can verify the following observations:

* every Conway game which is zero is also non-negative and non-positive,

* every Conway game which is non-positive and non-negative is also zero, 
* every Conway game which is negative is also non-positive and non zero,

* every Conway game which is non-positive and non zero is also negative,

* every Conway game which is positive is also non-negative and non zero,

* every Conway game which is non-negative and non zero is also positive,

* every Conway game which is fuzzy is also non non-negative and non non-positive, and

* every Conway game which is non non-negative and non non-positive is also fuzzy.

One can prove the following propositions:

(42) $g$ is zero, or positive, or negative, or fuzzy.

(43) $g$ is non-negative if and only if for every $g_{4}$ such that $g_{4} \in$ the right options of $g$ there exists $g_{8}$ such that $g_{8} \in$ the left options of $g_{4}$ and $g_{8}$ is non-negative.

(44) $g$ is non-positive if and only if for every $g_{7}$ such that $g_{7} \in$ the left options of $g$ there exists $g_{6}$ such that $g_{6} \in$ the right options of $g_{7}$ and $g_{6}$ is nonpositive.

(45)(i) $\quad g$ is non-negative iff for every $g_{4}$ such that $g_{4} \in$ the right options of $g$ holds $g_{4}$ is fuzzy or positive, and

(ii) $g$ is non-positive iff for every $g_{7}$ such that $g_{7} \in$ the left options of $g$ holds $g_{7}$ is fuzzy or negative.

(46) $g$ is fuzzy if and only if the following conditions are satisfied:

(i) there exists $g_{7}$ such that $g_{7} \in$ the left options of $g$ and $g_{7}$ is non-negative, and

(ii) there exists $g_{4}$ such that $g_{4} \in$ the right options of $g$ and $g_{4}$ is nonpositive.

(47) $g$ is zero if and only if the following conditions are satisfied:

(i) for every $g_{7}$ such that $g_{7} \in$ the left options of $g$ holds $g_{7}$ is fuzzy or negative, and

(ii) for every $g_{4}$ such that $g_{4} \in$ the right options of $g$ holds $g_{4}$ is fuzzy or positive.

(48) $g$ is positive if and only if the following conditions are satisfied:

(i) for every $g_{4}$ such that $g_{4} \in$ the right options of $g$ holds $g_{4}$ is fuzzy or positive, and

(ii) there exists $g_{7}$ such that $g_{7} \in$ the left options of $g$ and $g_{7}$ is non-negative.

(49) $g$ is negative if and only if the following conditions are satisfied:

(i) for every $g_{7}$ such that $g_{7} \in$ the left options of $g$ holds $g_{7}$ is fuzzy or negative, and

(ii) there exists $g_{4}$ such that $g_{4} \in$ the right options of $g$ and $g_{4}$ is nonpositive. 
One can check that $\mathbf{0}$ is zero.

Let us observe that $\mathbf{1}$ is positive and $*$ is fuzzy.

One can verify the following observations:

* there exists a Conway game which is zero,

* there exists a Conway game which is positive, and

* there exists a Conway game which is fuzzy.

Let $g$ be a non-positive Conway game. Note that $-g$ is non-negative.

Let $g$ be a non-negative Conway game. Note that $-g$ is non-positive.

Let $g$ be a positive Conway game. One can verify that $-g$ is negative.

Let us note that there exists a Conway game which is negative.

Let $g$ be a negative Conway game. Note that $-g$ is positive.

Let $g$ be a fuzzy Conway game. Note that $-g$ is fuzzy.

\section{REFERENCES}

[1] Grzegorz Bancerek. Cardinal numbers. Formalized Mathematics, 1(2):377-382, 1990.

[2] Grzegorz Bancerek. The fundamental properties of natural numbers. Formalized Mathematics, 1(1):41-46, 1990.

[3] Grzegorz Bancerek. The ordinal numbers. Formalized Mathematics, 1(1):91-96, 1990.

[4] Grzegorz Bancerek and Krzysztof Hryniewiecki. Segments of natural numbers and finite sequences. Formalized Mathematics, 1(1):107-114, 1990.

[5] Czesław Byliński. Functions and their basic properties. Formalized Mathematics, 1(1):55$65,1990$.

[6] J. H. Conway. On numbers and games. A K Peters Ltd., Natick, MA, second edition, 2001.

[7] Agata Darmochwał. Finite sets. Formalized Mathematics, 1(1):165-167, 1990.

[8] Zinaida Trybulec. Properties of subsets. Formalized Mathematics, 1(1):67-71, 1990.

[9] Edmund Woronowicz. Relations and their basic properties. Formalized Mathematics, 1(1):73-83, 1990.

Received October 13, 2010 